\title{
A machine learning algorithm to differentiate bipolar disorder from major depressive disorder using an online mental health questionnaire and blood biomarker data
}

Jakub Tomasik [1]', Sung Yeon Sarah Han', Giles Barton-Owen², Dan-Mircea Mirea ${ }^{1,5}$, Nayra A. Martin-Key',

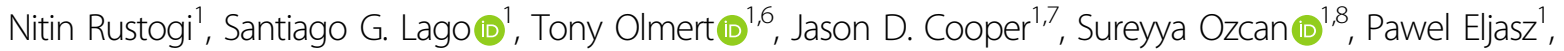

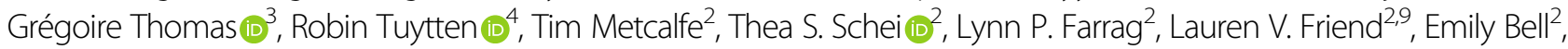
Dan Cowell ${ }^{2}$ and Sabine Bahn ${ }^{1,2}$

\begin{abstract}
The vast personal and economic burden of mood disorders is largely caused by their under- and misdiagnosis, which is associated with ineffective treatment and worsening of outcomes. Here, we aimed to develop a diagnostic algorithm, based on an online questionnaire and blood biomarker data, to reduce the misdiagnosis of bipolar disorder (BD) as major depressive disorder (MDD). Individuals with depressive symptoms (Patient Health Questionnaire-9 score 25) aged 18-45 years were recruited online. After completing a purpose-built online mental health questionnaire, eligible participants provided dried blood spot samples for biomarker analysis and underwent the World Health Organization World Mental Health Composite International Diagnostic Interview via telephone, to establish their mental health diagnosis. Extreme Gradient Boosting and nested cross-validation were used to train and validate diagnostic models differentiating BD from MDD in participants who self-reported a current MDD diagnosis. Mean test area under the receiver operating characteristic curve (AUROC) for separating participants with BD diagnosed as MDD $(N=126)$ from those with correct MDD diagnosis $(N=187)$ was $0.92(95 \% \mathrm{Cl}: 0.86-0.97)$. Core predictors included elevated mood, grandiosity, talkativeness, recklessness and risky behaviour. Additional validation in participants with no previous mood disorder diagnosis showed AUROCs of 0.89 (0.86-0.91) and 0.90 (0.87-0.91) for separating newly diagnosed $B D(N=98)$ from MDD $(N=112)$ and subclinical low mood $(N=120)$, respectively. Validation in participants with a previous diagnosis of $\mathrm{BD}(\mathrm{N}=45)$ demonstrated sensitivity of $0.86(0.57-0.96)$. The diagnostic algorithm accurately identified patients with $\mathrm{BD}$ in various clinical scenarios, and could help expedite accurate clinical diagnosis and treatment of BD.
\end{abstract}

Correspondence: Jakub Tomasik (jt455@cam.ac.uk) or

Sabine Bahn (sb209@cam.ac.uk)

${ }^{1}$ Department of Chemical Engineering and Biotechnology, University of

Cambridge, Cambridge, UK

${ }^{2}$ Psyomics Ltd, Cambridge, UK

Full list of author information is available at the end of the article

\section{Introduction}

Mood disorders are devastating psychiatric conditions which impose substantial burdens to individuals, healthcare systems and economies. Major depressive disorder (MDD) and bipolar disorder (BD) are two of the most common mood disorders and affect $\sim 16.6 \%$ and $3.9 \%$ of the global population, respectively, throughout their lifetime $^{1}$. In 2017 alone, about 163 million people $(2.1 \%$ of

\section{(c) The Author(s) 2021}

(c) (i) Open Access This article is licensed under a Creative Commons Attribution 4.0 International License, which permits use, sharing, adaptation, distribution and reproduction cc) in any medium or format, as long as you give appropriate credit to the original author(s) and the source, provide a link to the Creative Commons license, and indicate if changes were made. The images or other third party material in this article are included in the article's Creative Commons license, unless indicated otherwise in a credit line to the material. If material is not included in the article's Creative Commons license and your intended use is not permitted by statutory regulation or exceeds the permitted use, you will need to obtain permission directly from the copyright holder. To view a copy of this license, visit http://creativecommons.org/licenses/by/4.0/. 
global population) suffered from MDD and 46 million (0.6\%) were affected by BD, accounting for 32.8 million years lived with disability (YLDs) in the case of MDD and 9.3 million YLDs for $\mathrm{BD}^{2}$. These numbers have been steadily increasing since the $1990 \mathrm{~s}^{2}$ and both conditions are currently among the 20 leading causes of disability worldwide, with MDD ranked 2nd and BD 17th. In England, the direct economic burden of managing mood disorders, encompassing healthcare, informal care and justice system services, is estimated at $£ 1.68$ billion annually for depression and $£ 1.64$ billion for bipolar spectrum disorders, while indirect costs associated with lost work productivity amount to $£ 5.82$ billion and $£ 3.57$ billion, respectively, and are expected to grow ${ }^{4}$.

A large proportion of this burden is caused by incorrect or late diagnosis and treatment of $\mathrm{BD}$ and $\mathrm{MDD}^{5}$, and could be significantly reduced by means of early interventions ${ }^{4}$. Although BD can be distinguished from MDD by the intermittent occurrence of manic (BD I) or hypomanic (BD II) episodes, these often remain undiagnosed as patients are more likely to seek medical help during a depressive episode ${ }^{6}$. In turn, because depressive episodes in $\mathrm{BD}$ are indistinguishable from those in MDD, BD is often misdiagnosed as MDD, even if the depressive symptoms were preceded by a manic/hypomanic episode. In fact, $\sim 37 \%$ of patients with BD who present after their first manic/hypomanic episode are nonetheless misdiagnosed as having $\mathrm{MDD}^{7}$. Overall, it is estimated that at least $19 \%$ of individuals experiencing a major depressive episode have $\mathrm{BD}^{8}$, and that $\sim 40 \%$ of patients with $\mathrm{BD}$ are initially diagnosed with $\mathrm{MDD}^{7,9}$, with the average delay in $\mathrm{BD}$ diagnosis ranging from 5.7 to 7.5 years ${ }^{10,11}$. As a result, misdiagnosed patients with $\mathrm{BD}$ are often incorrectly treated with antidepressants, which can aggravate the disease and worsen the outcomes ${ }^{12}$.

The correct diagnosis of $\mathrm{BD}$ and $\mathrm{MDD}$ is further impeded by the unknown aetiology of these conditions and the lack of objective diagnostic measures. Diagnosing $\mathrm{BD}$ and MDD relies primarily on assessing patient selfreported symptoms in accordance to state-of-the-art diagnostic manuals, such as the Diagnostic and Statistical Manual of Mental Disorders, 5th Edition (DSM-5) ${ }^{13}$ or the International Statistical Classification of Diseases and Related Health Problems, 11th Revision (ICD-11) ${ }^{14}$. While structured psychiatric interviews are considered a gold standard for mental health disorder diagnosis, their systematic use in primary care, where the majority of MDD diagnoses are made, can be hindered by factors such as availability of qualified staff, inter-rater variability and time constraints ${ }^{15}$. In this regard, digital platforms offer a promising alternative for collecting and evaluating patient mental health data, while offering the advantage of being more easily available, adaptable, scalable and costeffective compared to traditional, interview-based methods ${ }^{16}$. Existing digital mental health applications are generally considered safe $^{17}$, although, despite their rapidly growing numbers, little evidence is available on their accuracy or efficacy ${ }^{16}$. Furthermore, it is anticipated that diagnostic accuracy in mental healthcare can be improved by incorporating biomarker profiling strategies, which could additionally provide a biological basis for mood disorder stratification and personalised treatment ${ }^{18}$.

We aimed to establish and validate a diagnostic algorithm, based on a new online mental health questionnaire and blood biomarker data, to detect $\mathrm{BD}$ in patients with a recent diagnosis of MDD and, hence, reduce the misdiagnosis of $\mathrm{BD}$ as MDD.

\section{Materials and methods}

\section{Study design and participants}

Data analysed here were collected as part of the Delta Study, an investigator-led study conducted by the Cambridge Centre for Neuropsychiatric Research (CCNR) at the University of Cambridge, which aimed to improve mood disorder diagnosis in participants presenting with depressive symptoms ${ }^{19-22}$. The primary objective of the Delta Study was to identify BD patients among patients who have recently ( $\leq 5$ years $)^{10,11}$ been diagnosed as having MDD. The study was approved by the University of Cambridge Human Biology Research Ethics Committee (approval number HBREC 2017.11) and was conducted in compliance with the Declaration of Helsinki ${ }^{23}$, Good Clinical Practice and ISO 14155:2011. A detailed research protocol for the Delta Study has been published previously $^{19}$. Participants were recruited online through email, via the CCNR website and Facebook. Inclusion criteria for the study required participants to be between 18 and 45 years old, residents of the United Kingdom, at least mildly depressed (Patient Health Questionnaire-9 ${ }^{24}$ total score 25 ), not pregnant or breastfeeding, and not suicidal. All participants read the participant information sheet and digitally provided informed consent for participation in the study. Recruitment started on 27 April 2018 and was completed on 28 September 2018. The current work complies with the Standards for Reporting of Diagnostic Accuracy Studies (STARD) ${ }^{25}$ and the Strengthening the Reporting of Observational Studies in Epidemiology (STROBE) ${ }^{26}$ guidelines.

\section{Procedures}

Upon enrolment, participants were asked to complete a purpose-built online mental health questionnaire available through the Delta Study website. The questionnaire was developed in collaboration with experienced psychiatrists and a service user advisory group and was based on existing structured diagnostic interviews as well as a range of mental health screening questionnaires ${ }^{19}$. It consisted of 635 distinct questions belonging to 6 
modules: (1) demographic information, (2) manic and hypomanic symptoms, (3) depressive symptoms, (4) personality traits, (5) psychiatric history and (6) other psychiatric conditions. The questionnaire was adaptive to answers given by participants, so that only relevant questions were asked, and the maximum possible number of questions asked to an individual was 382 (284 on average). Data collected from the questionnaire were used to identify participants qualifying for the study objectives, and as independent variables in statistical modelling.

Next, eligible participants who consented to providing a blood sample and completing a telephone diagnostic interview, who were free from blood-borne illnesses and had no previous diagnosis of schizophrenia, were provided with a dried blood spot (DBS) collection kit by post. The kit was designed to allow minimally invasive blood sample collection in a non-clinical setting, and was a Conformité Européenne-marked device under Article 22 of the Medical Device Regulation 2017/745. The kit included pre-injection cleaning swabs, sterile finger prick lancets, a DBS collection card (226 Spot Saver Cards, PerkinElmer), adhesive plasters and cotton pads. Detailed instructions for DBS sample collection were provided in a leaflet and as an online video. Participants were asked to spot 5 separate DBSs onto the card, after at least $6 \mathrm{~h}$ of fasting, and allow the card to dry for a minimum of $3 \mathrm{hrs}$ at room temperature. Cards were subsequently placed in the provided resealable bags with desiccant, and returned by post using pre-paid envelopes.

The returned DBS samples were analysed for neuropsychiatric biomarker levels using a validated targeted proteomic approach ${ }^{27-29}$. The method targeted 203 unique peptides representing 120 proteins (Supplementary Table 1) selected based on their association with psychiatric conditions and concentration in the blood ${ }^{28}$. DBS samples were processed using an automated Biomek NX workstation (Beckman Coulter). Proteins were extracted from $3 \mathrm{~mm}$ DBS discs using $50 \mathrm{mM}$ ammonium bicarbonate, followed by disulphide bond reduction with $5 \mathrm{mM}$ dithiothreitol and cysteine alkylation using $10 \mathrm{mM}$ iodoacetamide. Next, proteins were digested overnight with trypsin at 1:20 enzyme to protein ratio, followed by peptide purification in FNSC18 plates (Glygen Corp.) and elution with $60 \%$ acetonitrile. Stable isotope-labelled internal standard (SIS) peptides were subsequently spiked in for each target peptide to enable quantitative analysis. Infinity 1290 liquid chromatography system (Agilent) was used to separate $\sim 3.2 \mu \mathrm{g}$ of digested proteins on a $2.1 \times 150 \mathrm{~mm}$ AdvanceBio Peptide Mapping column (Agilent) at $50{ }^{\circ} \mathrm{C}$. Peptides were eluted using a gradient of acetonitrile in $0.1 \%$ formic acid from 3 to $30 \%$ over 45 minutes at $0.3 \mathrm{ml} / \mathrm{min}$, and analysed with a triple quadrupole mass spectrometer model 6495 (Agilent) equipped with a Jet Stream ion source operated in positive ionisation mode, using dynamic multiple reaction monitoring $^{27}$. Samples were randomised across plates, plate positions and experimental days to minimise technical bias, and quality control samples were included to monitor variation in sample preparation and instrument performance. Experimenters were blind to sample diagnostic allocation.

\section{Outcomes}

Participants who successfully completed the online questionnaire and returned the DBS sample were invited to complete the World Health Organization World Mental Health Composite International Diagnostic Interview (CIDI), version $3.0^{30}$ via telephone. The CIDI is a modular diagnostic tool which is widely used in epidemiological studies on mental health ${ }^{31}$ and shows good concordance with structured diagnostic interviews conducted by clinicians ${ }^{32}$. All interviewers conducting the CIDI received in-person training from an external CIDIcertified instructor, and internal training and mentoring. Only modules of the CIDI required for the lifetime mood disorder diagnosis, i.e. the screening, depression and mania sections, were implemented. We adopted voluntary response sampling, whereby the CIDI interviews continued until pre-specified study recruitment targets were met.

\section{Statistical analysis}

Power calculations for the study's primary objective showed that, assuming at least $80 \%$ sensitivity of the algorithm in detecting BD previously diagnosed as MDD and at least a $20 \%$ prevalence of $\mathrm{BD}$ among participants recently diagnosed with $\mathrm{MDD}^{8}$, a minimum of 200 participants with a recent diagnosis of MDD by a medical professional were required to provide at least $80 \%$ power to detect model noninferiority against an AUROC of 0.80 at the $5 \%$ significance level. The required number of participants was increased to 300 to account for potential dropouts, as observed in previous studies. Analogous calculations demonstrated that at least 300 symptomatic participants with no baseline diagnosis of mood disorder were required for the study's secondary objectives ${ }^{19}$. Additionally, we aimed to recruit 40 participants with a previous diagnosis of $\mathrm{BD}$ by a medical professional to validate the algorithm.

Data processing and analysis were conducted in $\mathrm{R}$ version $3.6 .3^{33}$. The online mental health questionnaire data were restructured so that answers to equivalent questions were concatenated (e.g. current and past symptoms), missing values were imputed where feasible (e.g. the number of relatives with depression was set to 0 for participants with no family history of mental health conditions), and features derived from the original variables were added (guided by the design of existing 
diagnostic algorithms, e.g. the number of symptoms). Ordinal questionnaire data were converted to ranks, and categorical data were encoded as dummy variables. Features that were duplicated, bijections or constant were removed. All missing values in the dataset were due to the adaptive character of the questionnaire (missing not at random and more likely for MDD). Raw biomarker data were processed in Skyline version 3.1.0 ${ }^{34}$. Peptides which were not detected $(N=9)$ were excluded from the analyses. Relative biomarker quantification was based on the ratios of abundances of the endogenous peptides over the abundances of the corresponding SIS peptides. Potential batch effects, caused by processing and analysing DBS samples across multiple plates, were adjusted for by median scaling. Biomarker level values were $\log _{2}$-transformed prior to analysis. The final number of analysed features was 1151, including 957 items from the online mental health questionnaire and 194 protein peptide measurements. The CIDI diagnosis was used as the dependent variable.

The diagnostic algorithm was trained and validated using data from participants with a recent self-reported diagnosis of MDD, confirmed as MDD or changed to $\mathrm{BD}$ by the CIDI. Participants whose DBS samples were not usable, or whose answers to the screening question about elevated mood on the online questionnaire and the telephone interview were inconsistent, were excluded (Fig. 1). Extreme Gradient Boosting (XGBoost) ${ }^{35}$, a decision treebased machine learning method, was selected to build the diagnostic algorithm, primarily because of its ability to handle missing values and detect non-linear relationships and interactions between variables, being robust to correlated features, as well as its interpretability. Nested cross-validation (CV), an equivalent to creating multiple train-test splits, was used to obtain robust estimates of model predictive performance in previously unseen data $^{36}$. We used 5-fold stratified nested CV, wherein at each iteration 4 of the folds were used in the inner loop to tune model parameters and train the algorithm, and the 5 th fold was used in the outer loop to test the trained model. Training of the XGBoost model was based on 5fold stratified CV repeated three times. Tuned model parameters included the number of trees (1 to 100), tree depth ( 1 or 2 , to allow for first order interactions) and the learning rate $(0.1$ or 0.3$)$. Initial testing showed that more extensive tuning was not required. Model performance was evaluated using AUROC. To avoid overfitting, optimal model parameters were defined as those which resulted in the simplest model with AUROC within 1 standard error below the AUROC of the best performing model. The final model was fitted using the tuned parameters to all data from the inner loop, and evaluated on the test set in the outer loop. Youden's J statistic ${ }^{37}$ was used to determine the optimal classification cut-off with balanced sensitivity and specificity. Nested CV was repeated 20 times, resulting in 100 models. The 95\% confidence intervals (CI) were estimated for all measures of diagnostic performance as the 2.5 th and 97.5 th percentiles across the 100 models. Features were evaluated based on their occurrence frequency across the 100 models and mean feature importance, i.e. gain (increase in accuracy brought by a feature to the branches it occurred on). The directionality of the relationship between the predictor and outcome variables was determined using the SHapley Additive exPlanations (SHAP) method ${ }^{38}$. The trained models were additionally validated in symptomatic participants with no previous diagnosis of mood disorder, and in participants with a previously established diagnosis of BD. Additional analyses included training classification models using: (1) subsamples of the training data, to assess potential bias related to sample size $\mathrm{e}^{39}$; (2) only features from the online questionnaire or only the biomarker data, to separately assess their potential utility; and (3) all available instances, i.e. including participants whose answers were inconsistent between the online and telephone assessments, to assess selection bias.

\section{Results}

The study flow diagram is shown in Fig. 1. To achieve study recruitment targets, 5422 symptomatic individuals were enrolled, of which 3232 completed the online mental health questionnaire, 1377 provided a DBS sample and 924 completed the CIDI diagnostic interview. The average time interval between starting the online assessment and completing the CIDI interview was 14 days. Only data from participants who returned a usable DBS sample and whose answers on the online questionnaire and the telephone interview were not inconsistent were analysed $(N=688$; Fig. 1$)$. These included 126 participants with $\mathrm{BD}$ $(N=76 \mathrm{BD}$ I and $50 \mathrm{BD}$ II) diagnosed as MDD and 187 participants with confirmed MDD from the primary dataset, and 98 newly diagnosed participants with $\mathrm{BD}$ ( $N=60 \mathrm{BD}$ I and $38 \mathrm{BD}$ II), 112 newly diagnosed participants with MDD, 120 participants with subclinical low mood (i.e. no previous mood disorder diagnosis and no mood disorder diagnosis from the CIDI), and 45 participants with a previous diagnosis of $\mathrm{BD}$ from the secondary datasets (Fig. 1). Demographic and clinical characteristics are shown in Table 1. Participants with BD who had been diagnosed as having MDD were, on average \pm standard deviation (SD), $27.4 \pm 7.2$ years old, $59 \%$ female, and overweight (BMI of $28.5 \pm 7.4$ ). The mean duration of MDD diagnosis in this group was $2.7 \pm 1.6$ years. The majority $(94 \%)$ had been treated with antidepressant medication, and the same percentage had never received any mood stabiliser treatment. Previous self-reported MDD diagnoses were made primarily by a General Practitioner (81.2\%), followed by those made by a 


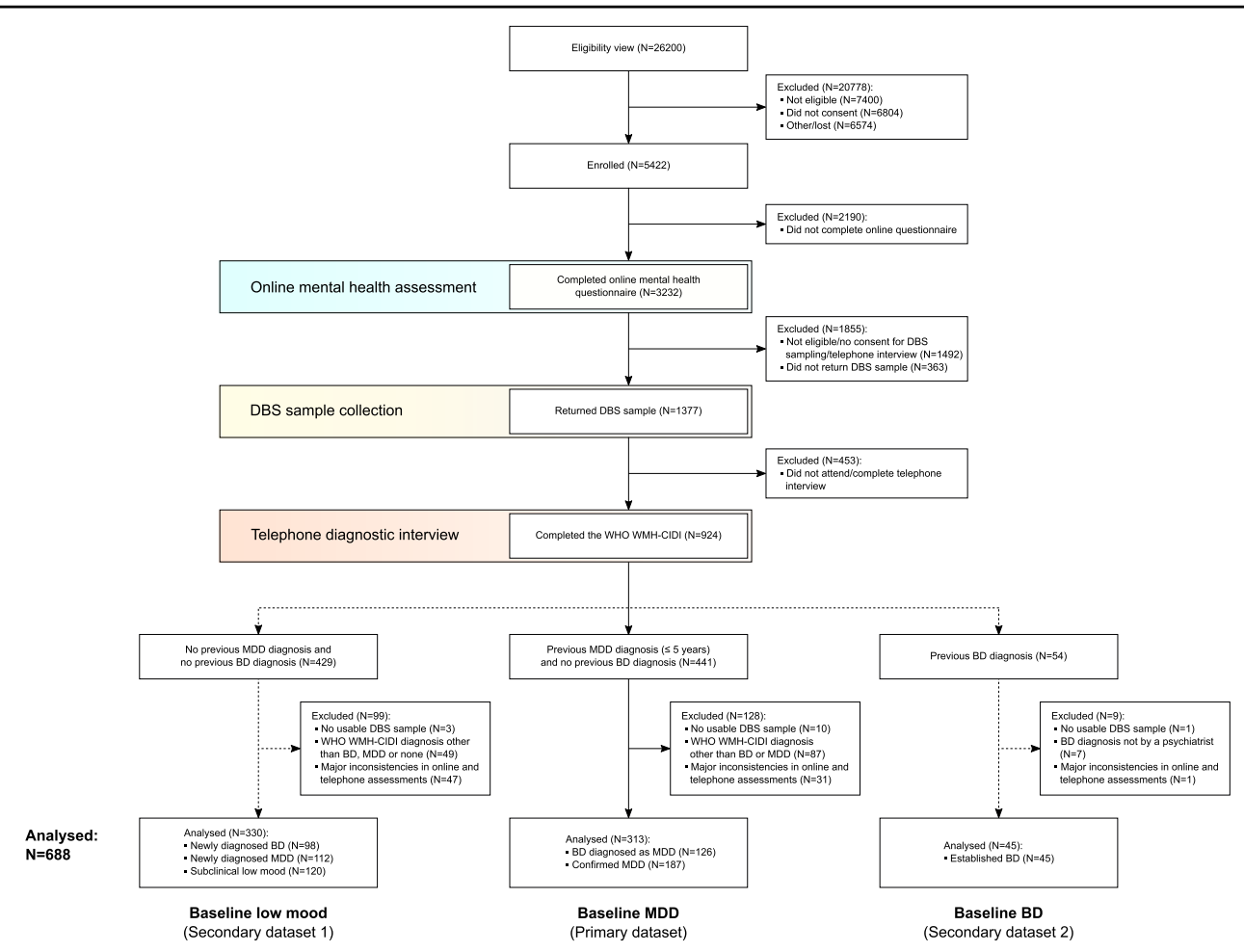

Fig. 1 Delta Study flow diagram. The diagram shows the number of individuals who completed each step of the study and reasons for attrition. BD bipolar disorder, DBS dried blood spot, MDD major depressive disorder, WHO WMH-CIDI World Health Organization World Mental Health Composite International Diagnostic Interview.

psychiatrist (18.5\%) and other medical professionals $(0.3 \%)$. Of the 45 participants with previously diagnosed $\mathrm{BD}, 35(78 \%)$ reported having been initially diagnosed with MDD, with the average time between MDD and BD diagnosis in this group being $5.5 \pm 5.9$ years. The mean duration of existing BD diagnosis was $7.5 \pm 6.8$ years.

The trained algorithms showed an out-of-fold AUROC of 0.92 (95\% CI: 0.86-0.97) in separating participants with $\mathrm{BD}$ previously diagnosed as MDD from those with confirmed MDD (Fig. 2A). In subgroup analyses, the AUROC was higher in participants with BD I $(0.94 ; 0.88-0.98)$ than in participants with BD II $(0.88 ; 0.78-0.95)$. The out-offold area under the precision-recall curve (AUPRC) in the primary dataset was 0.85 (0.73-0.95; Fig. 2B). Detailed estimates of out-of-fold model performance are summarised in Table 2.

The median number of features across the models was 9 , with the interquartile range between 6 and 15. Performance was driven primarily by the 5 features present in the majority of the models, namely elevated mood, grandiose delusions, talkativeness, recklessness, and risky behaviour (Table 3). Among the 30 most frequently selected features, 26 were from the online questionnaire, including questions on mania/hypomania, emotional instability, psychiatric history and comorbidities, and quality of life, and four were biomarker measurements.
Directionality of the relationships is shown in Supplementary Fig. 1. Follow-up analyses showed that models built using the online questionnaire or blood biomarker data separately had respective AUROCs of 0.92 (95\% CI: $0.85-0.97)$ and $0.50(0.34-0.62)$. Details of the models trained using only the online questionnaire data or the biomarker data are shown is Supplementary Tables 2-5.

Additional validation in the secondary datasets showed that the models separated participants with newly diagnosed BD from those with newly diagnosed MDD and subclinical low mood with respective AUROCs of 0.89 (95\% CI: 0.86-0.91) and $0.90(0.87-0.91)$, and AUPRCs of $0.87(0.81-0.90)$ and 0.84 (0.80-0.88; Fig. $2 \mathrm{C}-\mathrm{F}$ and Table 2). Furthermore, the models predicted the correct diagnosis in $86 \%$ (57-96\%) of participants with an established diagnosis of BD (Table 2).

Sensitivity analyses showed that the study was sufficiently powered, as indicated by the plateauing model performance at training set sizes $\geq 100$ (Supplementary Figs. 2 and 3). An additional analysis indicated that some selection bias might have been introduced when excluding participants who gave inconsistent answers on the online and telephone assessments. Including those participants in the analysis returned a test AUROC of 0.86 (95\% CI: 0.78-0.93) when distinguishing participants with $\mathrm{BD}$ previously diagnosed as MDD from those with confirmed MDD. 
Table 1 Demographic and clinical characteristics of study participants.

\begin{tabular}{|c|c|c|c|c|c|c|c|}
\hline \multirow[b]{2}{*}{ Diagnosis } & \multicolumn{2}{|c|}{ Baseline MDD } & \multicolumn{3}{|c|}{ Baseline low mood } & \multirow{2}{*}{$\begin{array}{l}\text { Baseline BD } \\
\text { BD }\end{array}$} & \multirow[b]{2}{*}{$P$ value } \\
\hline & $\mathrm{BD}$ & MDD & BD & MDD & Low mood & & \\
\hline N & 126 & 187 & 98 & 112 & 120 & 45 & NA \\
\hline Age, mean (SD), years & $27.4(7.2)$ & $28.1(6.9)$ & $25.4(5.9)$ & $26.4(6.2)$ & $25.8(6.5)$ & $33.9(7.8)$ & $<0.001$ \\
\hline \multicolumn{8}{|l|}{ Sex, $N(\%)$} \\
\hline Male & $52(41)$ & $50(27)$ & $36(37)$ & $30(27)$ & $40(33)$ & $28(62)$ & \multirow[t]{2}{*}{$<0.001$} \\
\hline Female & $74(59)$ & $137(73)$ & $62(63)$ & $82(73)$ & $80(67)$ & $17(38)$ & \\
\hline $\mathrm{BMI}$, mean $(\mathrm{SD}), \mathrm{kg} / \mathrm{m}^{2}$ & $28.5(7.4)$ & $28.3(7.0)$ & $26.3(6.6)$ & $26.6(6.6)$ & $24.6(4.5)$ & $28.6(6.2)$ & $<0.001$ \\
\hline \multicolumn{8}{|l|}{ Ethnicity, N (\%) } \\
\hline Asian/British Asian & $1(1)$ & $3(2)$ & $2(2)$ & $1(1)$ & $7(6)$ & $0(0)$ & \multirow[t]{7}{*}{0.104} \\
\hline Black/Black British & $1(1)$ & $0(0)$ & $0(0)$ & $0(0)$ & $0(0)$ & $0(0)$ & \\
\hline White & $93(74)$ & $132(71)$ & $61(62)$ & $74(66)$ & $73(61)$ & $37(82)$ & \\
\hline Mixed & $5(4)$ & $5(3)$ & $3(3)$ & $5(4)$ & $3(3)$ & $0(0)$ & \\
\hline Other & $0(0)$ & $1(1)$ & $1(1)$ & $2(2)$ & $4(3)$ & $0(0)$ & \\
\hline Prefer not to say & $0(0)$ & $0(0)$ & $0(0)$ & $0(0)$ & $0(0)$ & $0(0)$ & \\
\hline Unknown $^{a}$ & $26(21)$ & $46(25)$ & $31(32)$ & $30(27)$ & $33(28)$ & $8(18)$ & \\
\hline \multicolumn{8}{|l|}{ Smoking, $N(\%)$} \\
\hline No & $40(32)$ & $116(62)$ & $36(37)$ & $60(54)$ & $67(56)$ & $18(40)$ & \multirow[t]{2}{*}{$<0.001$} \\
\hline Yes & $86(68)$ & $71(38)$ & $62(63)$ & $52(46)$ & $53(44)$ & $27(60)$ & \\
\hline \multicolumn{8}{|l|}{ Alcohol consumption, $N(\%)$} \\
\hline No & $36(29)$ & $45(24)$ & $19(19)$ & $16(14)$ & $14(12)$ & $16(36)$ & \multirow[t]{2}{*}{0.001} \\
\hline Yes & $90(71)$ & $142(76)$ & $79(81)$ & $96(86)$ & $106(88)$ & $29(64)$ & \\
\hline \multicolumn{8}{|l|}{ Recreational drug use, $N(\%)$} \\
\hline No & $71(56)$ & $131(70)$ & $41(42)$ & $69(62)$ & $71(59)$ & $33(73)$ & \multirow[t]{2}{*}{$<0.001$} \\
\hline Yes & $55(44)$ & $56(30)$ & $57(58)$ & $43(38)$ & $49(41)$ & $12(27)$ & \\
\hline \multicolumn{8}{|l|}{ Education, $N(\%)$} \\
\hline$<\mathrm{GCSE}$ & $3(2)$ & $0(0)$ & $4(4)$ & $2(2)$ & $1(1)$ & $0(0)$ & \multirow[t]{5}{*}{0.001} \\
\hline GCSE & $18(14)$ & $19(10)$ & $8(8)$ & $5(4)$ & $10(8)$ & $8(18)$ & \\
\hline A-level & $49(39)$ & $46(25)$ & $33(34)$ & $23(21)$ & $32(27)$ & $12(27)$ & \\
\hline Undergraduate degree & $37(29)$ & $79(42)$ & $33(34)$ & $48(43)$ & $57(48)$ & $19(42)$ & \\
\hline Postgraduate degree & $19(15)$ & $43(23)$ & $20(20)$ & $34(30)$ & $20(17)$ & $6(13)$ & \\
\hline \multicolumn{8}{|l|}{ Employment, N (\%) } \\
\hline Employed/self-employed & $81(64)$ & $111(59)$ & $62(63)$ & $68(61)$ & $68(57)$ & $22(49)$ & \multirow[t]{5}{*}{$<0.001$} \\
\hline Parental leave & $0(0)$ & $1(1)$ & $0(0)$ & $0(0)$ & $0(0)$ & $0(0)$ & \\
\hline Student & $28(22)$ & $50(27)$ & $22(22)$ & $37(33)$ & $47(39)$ & $9(20)$ & \\
\hline Retired & $0(0)$ & $1(1)$ & $0(0)$ & $0(0)$ & $0(0)$ & $2(4)$ & \\
\hline Unemployed & $17(13)$ & $24(13)$ & $14(14)$ & $7(6)$ & $5(4)$ & $12(27)$ & \\
\hline \multicolumn{8}{|l|}{ Relationship status, N (\%) } \\
\hline In a relationship & $80(63)$ & $114(61)$ & $57(58)$ & $81(72)$ & $81(68)$ & $30(67)$ & \multirow[t]{2}{*}{0.275} \\
\hline Single & $46(37)$ & $73(39)$ & $41(42)$ & $31(28)$ & $39(32)$ & $15(33)$ & \\
\hline
\end{tabular}


Table 1 continued

\begin{tabular}{|c|c|c|c|c|c|c|c|}
\hline \multirow[b]{2}{*}{ Diagnosis } & \multicolumn{2}{|c|}{ Baseline MDD } & \multicolumn{3}{|c|}{ Baseline low mood } & \multirow{2}{*}{$\begin{array}{l}\text { Baseline BD } \\
\text { BD }\end{array}$} & \multirow[b]{2}{*}{$P$ value } \\
\hline & BD & MDD & BD & MDD & Low mood & & \\
\hline \multicolumn{8}{|l|}{ Childhood trauma, $N(\%)$} \\
\hline No & $44(35)$ & $84(45)$ & $32(33)$ & $55(49)$ & $77(64)$ & $15(33)$ & \multirow[t]{3}{*}{$<0.001$} \\
\hline Yes & $81(64)$ & $102(55)$ & $65(66)$ & $53(47)$ & $39(32)$ & $29(64)$ & \\
\hline Prefer not to say & $1(1)$ & $1(1)$ & $1(1)$ & $4(4)$ & $4(3)$ & $1(2)$ & \\
\hline \multicolumn{8}{|l|}{ Family psychiatric history, N (\%) } \\
\hline No & $27(21)$ & $31(17)$ & $27(28)$ & $38(34)$ & $41(34)$ & $2(4)$ & \multirow[t]{2}{*}{$<0.001$} \\
\hline Yes & $99(79)$ & $156(83)$ & $71(72)$ & $74(66)$ & $79(66)$ & $43(96)$ & \\
\hline Duration of MDD diagnosis, mean (SD), years & $2.7(1.6)$ & $2.6(1.5)$ & NA & NA & NA & $5.5(5.9)^{\mathrm{b}}$ & 0.037 \\
\hline \multicolumn{8}{|l|}{ Antidepressant treatment, $N(\%)$} \\
\hline SSRI & $116(92)$ & $174(93)$ & $27(28)$ & $16(14)$ & $14(12)$ & $38(84)$ & \multirow[t]{5}{*}{$<0.001$} \\
\hline SNRI & $24(19)$ & $22(12)$ & $2(2)$ & $2(2)$ & $1(1)$ & $20(44)$ & \\
\hline TCA & $13(10)$ & $19(10)$ & $6(6)$ & $2(2)$ & $1(1)$ & $16(36)$ & \\
\hline Other & $15(12)$ & $17(9)$ & $3(3)$ & $3(3)$ & $2(2)$ & $8(18)$ & \\
\hline None & $7(6)$ & $9(5)$ & $67(68)$ & $91(81)$ & $104(87)$ & $5(11)$ & \\
\hline Duration of $\mathrm{BD}$ diagnosis, mean (SD), years & NA & NA & NA & NA & NA & $7.5(6.8)$ & NA \\
\hline \multicolumn{8}{|l|}{ Mood stabiliser treatment, $N(\%)$} \\
\hline No & $119(94)$ & $180(96)$ & $97(99)$ & $112(100)$ & $120(100)$ & $18(40)$ & \multirow[t]{2}{*}{$<0.001$} \\
\hline Yes & $7(6)$ & $7(4)$ & $1(1)$ & $0(0)$ & $0(0)$ & $27(60)$ & \\
\hline \multicolumn{8}{|l|}{ Psychiatric hospitalisation, N (\%) } \\
\hline No & $105(83)$ & $170(91)$ & $91(93)$ & $111(99)$ & $119(99)$ & $25(56)$ & \multirow[t]{2}{*}{$<0.001$} \\
\hline Yes & $21(17)$ & $17(9)$ & $7(7)$ & $1(1)$ & $1(1)$ & $20(44)$ & \\
\hline PHQ-9 score, mean (SD) & $16.2(4.7)$ & $13.8(4.8)$ & $14.3(4.7)$ & $12.9(4.7)$ & $9.9(3.7)$ & $13.1(4.4)$ & $<0.001$ \\
\hline WEMWBS score, mean (SD) & $33.0(7.5)$ & $35.1(7.3)$ & $35.2(7.4)$ & $36.8(7.3)$ & $42.0(7.0)$ & $36.5(6.7)$ & $<0.001$ \\
\hline \multicolumn{8}{|l|}{ Fasting at DBS collection } \\
\hline No & $2(2)$ & $1(1)$ & $4(4)$ & $0(0)$ & $2(2)$ & $2(4)$ & \multirow[t]{2}{*}{0.097} \\
\hline Yes & $124(98)$ & $186(99)$ & $94(96)$ & $112(100)$ & $118(98)$ & $43(96)$ & \\
\hline
\end{tabular}

$P$ values were obtained from the Kruskal-Wallis test for continuous variables and $X^{2}$ test for categorical variables.

$B D$ bipolar disorder, BMI body mass index, DBS dried blood spot, GCSC General Certificate of Secondary Education, MDD major depressive disorder, NA not applicable, $P H Q-9$ Patient Health Questionnaire-9, SD standard deviation, SNRI serotonin-norepinephrine reuptake inhibitor, SSRI selective serotonin reuptake inhibitor, TCA tricyclic antidepressant. WEMWBS Warwick-Edinburgh Mental Wellbeing Scale.

anformation on ethnicity was collected at 6 months follow-up, which was not completed by all participants.

buntil BD diagnosis $(N=35)$.

\section{Discussion}

The main aim of the present study was to develop a diagnostic algorithm, based on an online mental health questionnaire and blood biomarker data, to identify BD patients among recently diagnosed MDD patients. The trained models achieved an average test AUROC of 0.92, with a mean accuracy of 0.83 , representing a $38 \%$ improvement compared to the baseline accuracy of 0.60 , i.e. the proportion of correctly diagnosed patients with MDD in the primary dataset (187/313). While we pre- specified the AUROC threshold for clinical relevance at $0.80^{19}$, the obtained estimate of above 0.90 is considered 'excellent ${ }^{40}$ or 'almost perfect ${ }^{32}$ for mental health disorder diagnosis. The remaining discrepancy between the algorithm and the CIDI outcomes is not unexpected given general diagnostic uncertainty surrounding psychiatric conditions, whereby even the 'gold standard' measures disagree in a small number of cases ${ }^{41}$.

The present results confirmed self-reported elevated mood, grandiosity, talkativeness, and recklessness as core 

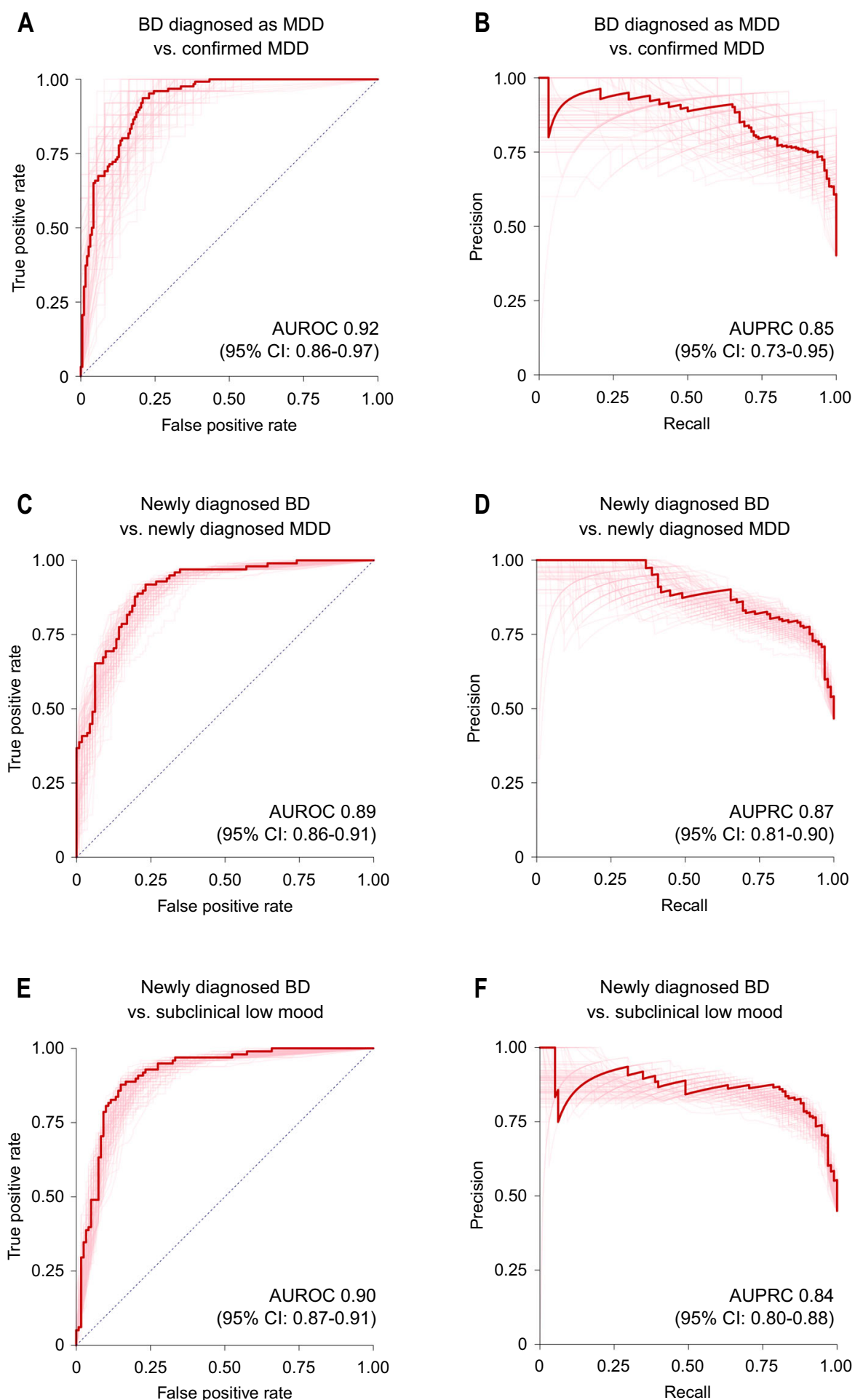

Fig. 2 Receiver operating characteristic and precision-recall curves for prediction of bipolar disorder diagnosis. A, B Out-of-fold results of nested cross-validation in the primary dataset ( $N=126$ BD previously diagnosed as MDD vs. $N=187$ confirmed MDD). Thick lines represent curves calculated from probabilities averaged across all models. C-F Validation in baseline low mood group $(N=98$ newly diagnosed BD vs. $N=112$ newly diagnosed MDD (C, D) and vs. N = 120 with subclinical depressive symptoms (E, F)). AUROC and AUPRC values represent mean ( $95 \%$ CI). AUPRC area under the precision-recall curve, AUROC area under the receiver operating characteristic curve, BD bipolar disorder, $\mathrm{Cl}$ confidence intervals, MDD major depressive disorder. 
Table 2 Out-of-fold model performance in the primary and secondary datasets.

\begin{tabular}{|c|c|c|c|c|}
\hline & \multirow{2}{*}{$\begin{array}{l}\text { Baseline MDD } \\
126 \text { BD vs. } 187 \text { MDD }\end{array}$} & \multicolumn{2}{|l|}{ Baseline low mood } & \multirow{2}{*}{$\begin{array}{l}\text { Baseline BD } \\
45 \text { BD }\end{array}$} \\
\hline & & 98 BD vs. 112 MDD & 98 BD vs. 120 low mood & \\
\hline AUROC & $0.92(0.86-0.97)$ & $0.89(0.86-0.91)$ & $0.90(0.87-0.91)$ & NA \\
\hline AUPRC & $0.85(0.73-0.95)$ & $0.87(0.81-0.90)$ & $0.84(0.80-0.88)$ & NA \\
\hline Accuracy & $0.83(0.76-0.91)$ & $0.80(0.75-0.84)$ & $0.82(0.77-0.86)$ & NA \\
\hline Sensitivity & $0.84(0.66-1.00)$ & $0.77(0.61-0.90)$ & $0.77(0.61-0.90)$ & $0.86(0.57-0.96)$ \\
\hline Specificity & $0.83(0.65-0.95)$ & $0.83(0.76-0.92)$ & $0.86(0.77-0.94)$ & NA \\
\hline PPV & $0.77(0.64-0.91)$ & $0.80(0.76-0.88)$ & $0.83(0.76-0.89)$ & NA \\
\hline NPV & $0.89(0.79-1.00)$ & $0.81(0.72-0.90)$ & $0.83(0.74-0.91)$ & NA \\
\hline
\end{tabular}

Values are shown as mean (95\% confidence intervals).

$A U P R C$ area under the precision-recall curve, $A U R O C$ area under the receiver operating characteristic curve, $B D$ bipolar disorder, $M D D$ major depressive disorder, $N A$ not applicable, NPV negative predictive value, PPV positive predictive value.

features of BD. Therefore, it is feasible that a simple, lowcost and highly scalable digital self-reporting tool could help expedite a correct diagnosis of BD by early capturing of emerging symptoms in patients presenting with depressive symptoms. The adaptive design of the questionnaire could be further streamlined by applying iterative machine learning algorithms, such as Bayesian updating or reinforcement learning, to offer dynamic question selection personalised to individual users. However, this approach would require a substantially larger training set size and limit the amount of user data available for future exploratory analyses. Introducing such digital instruments into primary healthcare, where resources are scarce and where symptoms of $\mathrm{BD}$ often remain undiagnosed ${ }^{42}$, has the potential to lessen the burden experienced by both patients and medical professionals, and therefore reduce the overall load on the healthcare system. In particular, such an approach could constitute a cost- and time-effective alternative to conventional, interview-based methods, while allowing for a more comprehensive symptom assessment and identification of patients who require specialty care services early in the mental health triage process.

In addition, among the top ranked predictors were more objective features such as symptoms reported as being 'observed by others' concerning risky behaviour and speaking faster, the number of second-degree relatives with MDD, sleep disturbances, and several biomarkers including kininogen-1 (KNG1) and thrombospondin-1 (TSP1), proteins previously reported to discriminate $\mathrm{BD}$ from $\mathrm{MDD}^{43,44}$. Although biomarker data alone were not predictive of the disease status, their selection alongside digital features in some models suggests their potential utility in subgroups of patients or in specific symptom contexts. All together, these results indicate the emerging potential for more objective and systematic diagnostic approaches, such as digital phenotyping of symptoms ${ }^{45}$, multi-reporter assessment systems ${ }^{46}$, and genetic ${ }^{47}$ and proteomic ${ }^{44,48}$ biomarker profiling, in aiding the diagnosis of BD.

This study has a number of advantages compared to previous studies aiming to distinguish BD from MDD during depressive episodes. To our knowledge it is the largest investigation, with more than twice as many participants as the largest study to date $(N=313$ vs. $N=$ $112)^{44}$. It has also been more extensively validated, through the application of nested CV in the primary dataset and additional testing in two secondary datasets, while previous studies employed 10-fold or leave-one-out $\mathrm{CV}$ and no external test sets. Although not directly comparable, the algorithm outperformed existing models, for which the maximum AUROC was $0.9058^{49}$. The current study is also unique in its aim to develop a robust heuristic algorithm to detect BD in individuals with a recent diagnosis of MDD based on combined symptom and biomarker data.

The present results should be interpreted within their limitations. Due to recruiting participants through the internet, and in order to meet specific study recruitment targets, the analysed population might be biased and not representative of patients presenting in primary or secondary care services. In addition, we did not have access to participants' medical records and could not verify the self-reported psychiatric history. Also, despite attempting to control for the consistency of answers between the online and telephone assessments, other inaccuracies might have remained in the dataset. It is also important to note that such step would be unfeasible in real-life applications, and alternative approaches, built into the online questionnaire, should be employed to protect data integrity $^{50}$. Finally, BD might have not been detected in participants who have not yet experienced a manic/ hypomanic episode, and a longitudinal study would be 
Table 3 Top predictors of bipolar disorder.

\begin{tabular}{|c|c|c|c|}
\hline Feature & Frequency & Importance, mean (SD) & Category \\
\hline Elevated mood & 0.99 & $0.182(0.080)$ & Bipolar/hypomania \\
\hline Grandiosity & 0.87 & $0.095(0.042)$ & Bipolar/hypomania \\
\hline More talkative & 0.82 & $0.193(0.089)$ & Bipolar/hypomania \\
\hline Recklessness (HMQ) & 0.82 & $0.122(0.087)$ & Bipolar/hypomania \\
\hline Others: risky behaviour & 0.77 & $0.232(0.122)$ & Bipolar/hypomania \\
\hline Others: speaking faster & 0.38 & $0.033(0.018)$ & Bipolar/hypomania \\
\hline Recklessness (PQ) & 0.38 & $0.041(0.016)$ & Emotional instability \\
\hline Increased energy & 0.37 & $0.055(0.030)$ & Bipolar/hypomania \\
\hline Mood lability & 0.35 & $0.037(0.013)$ & Emotional instability \\
\hline Risky behaviour & 0.31 & $0.225(0.125)$ & Bipolar/hypomania \\
\hline Episode duration & 0.30 & $0.050(0.021)$ & Bipolar/hypomania \\
\hline$\geq 3$ symptoms & 0.30 & $0.143(0.099)$ & Bipolar/hypomania \\
\hline Second-degree relatives with MDD & 0.27 & $0.030(0.014)$ & History/comorbidities \\
\hline Past elevated mood & 0.25 & $0.025(0.009)$ & Bipolar/hypomania \\
\hline Fear of abandonment & 0.22 & $0.031(0.013)$ & Emotional instability \\
\hline Number of episodes & 0.21 & $0.050(0.021)$ & Bipolar/hypomania \\
\hline Self-image instability & 0.18 & $0.034(0.014)$ & Emotional instability \\
\hline Racing thoughts & 0.14 & $0.065(0.045)$ & Bipolar/hypomania \\
\hline Feeling loved (2 weeks) & 0.14 & $0.022(0.008)$ & Quality of life \\
\hline KNG1 (YFIDFVAR) & 0.12 & $0.022(0.008)$ & Biomarker \\
\hline Unstable relationships & 0.12 & $0.023(0.006)$ & Emotional instability \\
\hline IGHG1 (FNWYVDGVEVHNAK) & 0.11 & $0.014(0.005)$ & Biomarker \\
\hline Recklessness (BDQ) & 0.10 & $0.040(0.016)$ & Bipolar/hypomania \\
\hline Functional impairment/Hospitalisation & 0.09 & $0.055(0.031)$ & Bipolar/hypomania \\
\hline TSP1 (GTLLALER) & 0.09 & $0.015(0.007)$ & Biomarker \\
\hline Social activity & 0.08 & $0.031(0.023)$ & Bipolar/hypomania \\
\hline APOA1 (ATEHLSTLSEK) & 0.07 & $0.008(0.008)$ & Biomarker \\
\hline Productivity & 0.07 & $0.017(0.008)$ & Bipolar/hypomania \\
\hline Sleep distress & 0.07 & $0.004(0.003)$ & History/comorbidities \\
\hline Duration of social phobia & 0.07 & $0.010(0.004)$ & History/comorbidities \\
\hline
\end{tabular}

Table shows 30 most frequently selected features and their importance (i.e. gain). Biomarkers are labelled as 'UniProtKB protein ID (target peptide sequence)'. $A P O A 1$ apolipoprotein $\mathrm{A} 1, B D Q$ bipolar disorder questionnaire, HMQ hypomania questionnaire, IGHG1 immunoglobulin heavy constant gamma 1, KNG1 kininogen 1 , $M D D$ major depressive disorder, $P Q$ personality disorder questionnaire, SD standard deviation, TSP1 thrombospondin-1, UniProtKB UniProt Knowledgebase.

required to determine the correct diagnosis for those individuals.

In conclusion, our study provides a proof of concept that an evidence-based algorithm can accurately detect $\mathrm{BD}$ in patients recently diagnosed with MDD. The results may generalise to other clinically relevant populations. Further work is required to rigorously assess the potential of incorporating such algorithms into primary healthcare, where the majority of MDD diagnoses are made, to expedite the diagnosis of $\mathrm{BD}$ and reduce workload for healthcare professionals.

\section{Acknowledgements}

This study was funded by the Stanley Medical Research Institute (grant number 07R-1888) and Psyomics Ltd. We are most grateful to all participants of the Delta Study for their generous contributions and making this work possible. We are also grateful to Sharmelee Thiahulan, Rhian L. Preece, Dr Mark Agius, Dr Neil Hunt, the CIDI interviewers, DBS kit packers and all members of the Delta Study Service User Advisory Group, for their valuable input in designing and conducting the study. 


\section{Author details}

${ }^{1}$ Department of Chemical Engineering and Biotechnology, University of Cambridge, Cambridge, UK. ${ }^{2}$ Psyomics Ltd, Cambridge, UK. ${ }^{3}$ SQU4RE, Roeselare, Belgium. ${ }^{4}$ Metabolomic Diagnostics, Little Island, Cork, Ireland. ${ }^{5}$ Present address: Princeton Neuroscience Institute, Princeton University, Princeton, New Jersey, USA. ${ }^{6}$ Present address: University of California San Diego School of Medicine, San Diego, California, USA. ${ }^{7}$ Present address: Owlstone Medical Ltd, Cambridge, UK. PPresent address: Department of Chemistry, Middle East Technical University, Ankara, Turkey. ${ }^{9}$ Present address: KPMG UK, London, UK

\section{Code availability}

Upon request from the corresponding author SB.

\section{Conflict of interest}

S.B. is a director of Psynova Neurotech Ltd and Psyomics Ltd. S.B., D.C., G.B.O., L. P.F. and E.B. have financial interests in Psyomics Ltd. S.B., P.E. and T.O. have received payments from the University of Cambridge for licensing of data from the Delta Study. All other authors declare no competing interests.

\section{Publisher's note}

Springer Nature remains neutral with regard to jurisdictional claims in published maps and institutional affiliations.

Supplementary Information accompanies this paper at (https://doi.org/ 10.1038/s41398-020-01181-x).

Received: 19 August 2020 Revised: 14 December 2020 Accepted: 15 December 2020

Published online: 12 January 2021

\section{References}

1. Kessler, R. C. et al. Lifetime prevalence and age-of-onset distributions of DSMIV disorders in the national comorbidity survey replication. Arch. Gen. Psychiatry 62, 593-602 (2005).

2. James, S. L. et al. Global, regional, and national incidence, prevalence, and years lived with disability for 354 Diseases and Injuries for 195 countries and territories, 1990-2017: A systematic analysis for the Global Burden of Disease Study 2017. Lancet 392, 1789-1858 (2018).

3. Vigo, D., Thornicroft, G. \& Atun, R. Estimating the true global burden of mental illness. Lancet Psychiatry 3, 171-178 (2016).

4. McCrone P., Dhanasiri S., Patel A., Knapp M., Lawton-Smith S. Paying the price: the cost of mental health care in England to 2026 (King's Fund, London, 2008).

5. Hall, R. C. W. \& Wise, M. G. The Clinical and financial burden of mood disorders: cost and outcome. Psychosomatics 36, S11-S18 (1995).

6. Hirschfeld, R. M. A., Cass, A. R., Holt, D. C. L. \& Carlson, C. A. Screening for bipolar disorder in patients treated for depression in a family medicine clinic. J. Am. Board Fam. Pract. 18, 233-239 (2005).

7. Ghaemi, S. N., Boiman, E. E. \& Goodwin, F. K. Diagnosing bipolar disorder and the effect of antidepressants: a naturalistic study. J. Clin. Psychiatry 61, 804-808 (2000).

8. Cassano, G. B., Akiskal, H. S., Savino, M., Musetti, L. \& Perugi, G. Proposed subtypes of bipolar II and related disorders: with hypomanic episodes (or cyclothymia) and with hyperthymic temperament. J. Affect Disord. 26, 127-140 (1992).

9. Hirschfeld, R. M. A., Lewis, L. \& Vornik, L. A. Perceptions and impact of bipolar disorder: How far have we really come? Results of the National Depressive and Manic-Depressive Association 2000 Survey of individuals with bipolar disorder. J. Clin. Psychiatry 64, 161-174 (2003).

10. Ghaemi, S. N., Sachs, G. S. M., Chiou, A., Pandurangi, A. K. \& Goodwin, F. K. Is bipolar disorder still underdiagnosed? Are antidepressants overutilized? J. Affect Disord. 52, 135-144 (1999).

11. Morselli, P. L. \& Elgie, R., GAMIAN-Europe. GAMIAN-Europe/BEAM Survey I-global Analysis of a Patient Questionnaire circulated to 3450 members of 12 European Advocacy Groups operating in the field of mood disorders. Bipolar Disord. 5, 265-278 (2003).
12. Pacchiarotti, I. et al. The International Society for bipolar Disorders (ISBD) task force report on antidepressant use in bipolar disorders. Am. J. Psychiatry 170, 1249-1262 (2013).

13. American Psychiatric Association. Diagnostic and Statistical Manual of Mental Disorders. 5th edn (Washington, DC, 2013).

14. World Health Organization. International Statistical Classification of Diseases and Related Health Problems. 11th edn. (WHO. 2019).

15. Cabana, M. D. et al. Why don't physicians follow clinical practice guidelines?: A framework for improvement. J. Am. Med. Assoc. 282, 1458-1465 (1999).

16. Weisel, K. K. et al. Standalone smartphone apps for mental health-a systematic review and meta-analysis. npj Digit. Med. 2, 118 (2019).

17. Husain, I. \& Spence, D. Can healthy people benefit from health apps? BMJ $\mathbf{3 5 0}$ h1887 (2015)

18. Phillips, M. L. \& Kupfer, D. J. Bipolar disorder diagnosis: challenges and future directions. Lancet 381, 1663-1671 (2013).

19. Olmert, T. et al. A combined digital and biomarker diagnostic aid for mood disorders (the Delta Trial): protocol for an observational study. JMIR Res. Protoc. 9, e18453 (2020).

20. Han, S. Y. S. et al. Diagnostic prediction model development using data from dried blood spot proteomics and a digital mental health assessment to identify major depressive disorder among individuals presenting with low mood. Brain Behav. Immun. 90, 184-195 (2020).

21. Martin-Key, N. A. et al. Advances in digital psychiatry - Towards an extended definition of major depressive disorder symptomatology. submitted 2020.

22. Mirea, D.-M. et al. The impact of an online psychiatric assessment on the mental health and wellbeing of participants presenting with depressive symptoms: longitudinal observational study. JMIR Mental Health https:/doi. org/10.2196/23813 (2020).

23. World Medical Association. World Medical Association Declaration of Helsinki: ethical principles for medical research involving human subjects. JAMA $\mathbf{3 1 0}$, 2191-2194 (2013).

24. Kroenke, K., Spitzer, R. L. \& Williams, J. B. W. The PHQ-9: Validity of a brief depression severity measure. J. Gen. Intern. Med. 16, 606-613 (2001).

25. Bossuyt, P. M. et al. STARD 2015: an updated list of essential items for reporting diagnostic accuracy studies. BMJ 351, h5527 (2015).

26. von Elm, E. et al. The Strengthening the Reporting of Observational Studies in Epidemiology (STROBE) statement: guidelines for reporting observational studies. Lancet 370, 1453-1457 (2007).

27. Ozcan, S. et al. Towards reproducible MRM based biomarker discovery using dried blood spots. Sci. Rep. 7, 45178 (2017).

28. Cooper, J. D. et al. Multimodel inference for biomarker development: an application to schizophrenia. Transl. Psychiatry 9, 83 (2019).

29. Han, S. Y. S. et al. Integrating proteomic, sociodemographic and clinical data to predict future depression diagnosis in subthreshold symptomatic individuals. Transl. Psychiatry 9, 277 (2019).

30. Kessler, R. C. \& Üstün, B. B. The World Mental Health (WMH) Survey Initiative version of the World Health Organization (WHO) Composite International Diagnostic Interview (CIDI). Int. J. Methods Psychiatr. Res. 13, 93-121 (2004).

31. Kessler, R. C., Wai, T. C., Demler, O. \& Walters, E. E. Prevalence, severity, and comorbidity of 12-month DSM-IV disorders in the National Comorbidity Survey Replication. Arch. Gen. Psychiatry 62, 617-627 (2005).

32. Haro, J. M. et al. Concordance of the Composite International Diagnostic Interview Version 3.0 (CIDI 3.0) with standardized clinical assessments in the WHO World Mental Health Surveys. Int J. Methods Psychiatr. Res. 15, 167-180 (2006).

33. R Core Team. R: A Language and Environment for Statistical Computing. https://www.r-project.org/ (2020).

34. MacLean, B. et al. Skyline: an open source document editor for creating and analyzing targeted proteomics experiments. Bioinformatics 26, 966-968 (2010).

35. Chen T., Guestrin C. XGBoost: a scalable tree boosting system. arXiv https://doi. org/10.1145/2939672.2939785 (2016).

36. Varma, S. \& Simon, R. Bias in error estimation when using cross-validation for model selection. BMC Bioinform. 7, 91 (2006).

37. Youden, W. J. Index for rating diagnostic tests. Cancer 3, 32-35 (1950).

38. Lundberg, S. M., Lee, S. I. A Unified Approach to Interpreting Model Predictions. Advances in Neural Information Processing Systems 30, 4765-4774 (2017).

39. Flint, C. et al. Systematic overestimation of machine learning performance in neuroimaging studies of depression. arXiv. https:/arxiv.org/abs/1912.06686 (2019).

40. Li, F. \& He, H. Assessing the Accuracy of Diagnostic Tests. Shanghai Arch. Psychiatry 30, 207-212 (2018). 
41. Kaltenboeck, A., Winkler, D. \& Kasper, S. Bipolar and related disorders in DSM-5 and ICD-10. CNS Spectr. 21, 318-323 (2016).

42. Hughes, T. et al. Unrecognised bipolar disorder among UK primary care patients prescribed antidepressants: an observational study. Br. J. Gen. Pract. 66, e71-e77 (2016).

43. Chen, J. et al. Comparative proteomic analysis of plasma from bipolar depression and depressive disorder: identification of proteins associated with immune regulatory. Protein Cell 6, 908-911 (2015).

44. Kittel-Schneider, S. et al. Proteomic profiling as a diagnostic biomarker for discriminating between bipolar and unipolar depression. Front. Psychiatry 11, 189 (2020).

45. Huckvale, K., Venkatesh, S. \& Christensen, H. Toward clinical digital phenotyping: a timely opportunity to consider purpose, quality, and safety. npj Digit. Med. 2, 88 (2019).
46. Pavlova B., Uher R. Assessment of psychopathology: is asking questions good enough? JAMA Psychiatry 77, 557-558 (2020).

47. Liebers, D., Pirooznia M., Ganna A., Goes F. Discriminating bipolar depression from major depressive disorder with polygenic risk scores. Psychol. Med. https://doi.org/10.1017/S003329172000015X (2020).

48. Haenisch, F. et al. Towards a blood-based diagnostic panel for bipolar disorder. Brain Behav. Immun. 52, 49-57 (2016).

49. Zheng, Y. et al. Detection study of bipolar depression through the application of a model-based algorithm in terms of clinical feature and peripheral biomarkers. Front. Psychiatry 10, 266 (2019).

50. Teitcher, J. E. F. et al. Detecting, preventing, and responding to 'fraudsters' in internet research: ethics and tradeoffs. J. Law Med. Ethics 43, 116-133 (2015) 